\title{
Simulation Analysis of a Multiple-Vehicle, High-Speed Train Collision Using a Simplified Model
}

\author{
Suchao Xie $\mathbb{I D}^{1,2}$ Weilin Yang, ${ }^{1,2}$ and Ping Xu (iD) ${ }^{1,2}$ \\ ${ }^{1}$ Key Laboratory of Traffic Safety on Track, Ministry of Education, Central South University, Changsha, Hunan 410075, China \\ ${ }^{2}$ School of Traffic \& Transportation Engineering, Central South University, Changsha, Hunan 410075, China \\ Correspondence should be addressed to Suchao Xie; xsc0407@csu.edu.cn
}

Received 12 October 2017; Revised 27 December 2017; Accepted 17 January 2018; Published 5 March 2018

Academic Editor: Yuri S. Karinski

Copyright (c) 2018 Suchao Xie et al. This is an open access article distributed under the Creative Commons Attribution License, which permits unrestricted use, distribution, and reproduction in any medium, provided the original work is properly cited.

\begin{abstract}
To solve the problems associated with multiple-vehicle simulations of railway vehicles including large scale modelling, long computing time, low analysis efficiency, need for high performance computing, and large storage space, the middle part of the train where no plastic deformation occurs in the vehicle body was simplified using mass and beam elements. Comparative analysis of the collisions between a single railway vehicle (including head and intermediate vehicles before, and after, simplification) and a rigid wall showed that variations in impact kinetic energy, internal energy, and impact force (after simplification) are consistent with those of the unsimplified model. Meanwhile, the finite element model of a whole high-speed train was assembled based on the simplified single-vehicle model. The numbers of nodes and elements in the simplified finite element model of the whole train were $63.4 \%$ and $61.6 \%$, respectively, compared to those of the unsimplified model. The simplified whole train model using the above method was more accurate than the multibody model. In comparison to the full-size finite element model, it is more specific, had more rapid computational speed, and saved a large amount of computational power and storage space. Finally, the velocity and acceleration data for every car were discussed through the analysis of the collision between two simplified trains at various speeds.
\end{abstract}

\section{Introduction}

With the development of railway transportation and the increases in train speeds, attention has been paid to the safety of train operations. Although the probability of a collision between railway vehicles is small, once it happens, significant losses will be induced due to the trains running at high speeds and high kinetic energy [1-3]. Although extensive studies have been conducted on the crashworthiness of trains, as characterised by their energy absorption, they merely focus on the car-body design of a single vehicle [4-9]. Research into crashworthiness, strength, and the vibrational features of an aluminium railroad passenger vehicle indicated that the stresses, natural frequencies, and crashworthiness were within acceptable tolerances, but the final design (in aluminium) was about one-third of the weight of the initial steel structure [10]. Two detailed modifying measures, that is, weakening the structure of the end of the floor and strengthening the structure of the cabin, had been adopted to improve crashworthiness by design [11].
A train comprises multiple vehicles. Differing from the single-body crash of a vehicle, train collision is usually the coupled collisions of multiple vehicles. When the two end trunks of two trains are in contact and then collide with each other, the vehicle thereafter is supposed to collide in sequence with the front vehicle [12-14]. Three-dimensional crash simulations of a high-speed train were conducted by using multibody dynamics, which showed that it was possible to simulate overriding, derailment, and lateral buckling, but several factors such as computational time and large deformation of the structures needed further investigation [13]. A validated multibody-based model was presented for the design of train crashworthy components, and the validated model was applied to the collision of two different trains [15]. It was shown that the simulation of the model with the design specifications, characterised by elastic-perfectly plastic deformation curves for the structural elements, led to the results similar to those observed experimentally. Milho et al. [16] proposed a multibody-based method for the study of train crashworthiness including anticlimber 
devices. The method was applied to train impacts in various crash scenarios, which were characterised by collisions with different velocities against stopped trains. The modelling assumptions and the suitability of the numerical tools developed were discussed in the framework of their application to the design of train crashworthy components. Kirkpatrick et al. [17] compared the various experimental, analytical, and computational approaches used to evaluate railway vehicle crashworthiness. Analyses ranged from simplified onedimensional models used to evaluate interactions between vehicles and study the effects of varying parameters, such as crushing strength, to detailed three-dimensional finite element crash simulations that could be used as part of the vehicle design process. Simons and Kirkpatrick [14] developed a mathematical method for the crashworthiness analyses of trains. The approach was organised in three steps: first, a detailed model of the train vehicle was developed and its response calculated for various collision scenarios; the second step used the crush response to develop simpler models that could be used to explore the collision dynamics between vehicles; the third step used the vehicle velocity histories to calculate the response of an occupant in the train.

Therefore, it can be seen that train collision is a complicated physical phenomenon. Apart from the coupled collision between vehicles, such collisions also involve geometric nonlinearity caused by large displacements and rotation of the vehicle body and physical nonlinearity, complicated impact and friction problems exhibited when various materials used in any vehicle are subject to large strains $[18,19]$. Numerical simulation is the main method used to investigate large deformation collisions. The methods commonly used in the analysis of numerical simulation include multibody dynamics and nonlinear finite element analysis. Multibody dynamics analyses the motion of the system and uses rigid, or flexible, bodies to represent the vehicle body, bogie, suspension parts, the structure of passenger compartment, crash-test dummies, and so on [20] while studying collisions between railway vehicles. Yan et al. [21] established a numerical model of a shield tunnel subjected to impact loads from a derailed train based on the finite element modelling strategy. The analysis showed compressive damage was mainly observed on the target and adjacent segments. Although it cannot obtain the impact induced deformation and stress and strain distributions within the vehicle structure, it is highly efficient while solving collision response values. The dynamic nonlinear finite element method (FEM) can describe the system using various elements and nodes and has been used in detailed modelling of vehicle structures [22]. As it is capable of providing detailed information regarding the structural deformation, the strength, and distribution of stress and strain over the whole vehicle body, it has therefore been widely applied in the analysis of vehicle collisions. Meran et al. [23] performed a full-scale finite element simulation of passenger cars and demonstrated the effectiveness of a crush zone in improving crashworthiness. They pointed out that modelling of the whole train set was necessary to evaluate and verify stability, the level of deceleration, wheel-lift, and the strength of the car body. However, when the nonlinear finite element method is used for large deformation dynamic analysis, much time is spent on the calculation, especially in the case of the collisions involving the whole train set.

Concerning studies on the finite element based crashworthiness prediction of railway vehicles, Tang et al. [24] proposed a data-driven train crash modelling method to improve computational speed. The method did not need FEM to calculate but refined the mechanical patterns from existing FE calculation results which were then combined with the multibody models to achieve a fast dynamic simulation and predict the crash appearance in different initial velocity. Afazov et al. [25] used a simplified illustration of the modelled train unit in which the length of the components was modelled by controlling the coordinates of the nodes connecting the truss elements and found that the simplified model could successfully be used at the concept design stage for crashworthiness testing. To construct a finite element model for the collision analysis of a whole train set consisting of multiple vehicles, the vehicle structure has to be simplified to some extent. This research mainly investigated such a simplified model of a train collision.

\section{Materials and Methods}

2.1. Model Simplification Methods. This research aimed to establish a finite element model for a high-speed train using LS-DYNA. The disadvantages such as large model scale, long computing time, low analysis efficiency, need for high computer performance, and large storage space are found in the multiple-vehicle simulation of railway vehicles. To solve the aforementioned problems, this research simplified the vehicle body by applying the finite element model of the vehicle body of a high-speed train as the research object. All collision simulations were performed using a DELL computer with the Windows Server R2 Enterprise operating system. The main specifications of the computer were an Intel Xeon 4 core CPU, $2.50 \mathrm{GHz}$ main frequency, $128 \mathrm{~GB}$ memory, and 1000 GB hard disk capacity. The detailed simplification steps were as follows:

(1) The analysis is performed on the collision between single vehicles which refer to head and intermediate railways vehicles and an ideal rigid wall at various speeds $(5 \mathrm{~m} / \mathrm{s}$, $7.5 \mathrm{~m} / \mathrm{s}, 10 \mathrm{~m} / \mathrm{s}$, and $15 \mathrm{~m} / \mathrm{s})$. Afterwards, the part where no plastic deformation occurred while restorable elastic deformation is allowed in the vehicle body is analysed, as shown in the middle part of Figure 1. Then, this part is extracted and its total mass and centre of gravity are calculated.

(2) The middle part of the vehicle body extracted in step (1) is replaced by mass elements $m_{1}$ and $m_{2}$. The positions of the mass elements $m_{1}$ and $m_{2}$ are the centres of gravity of the simplified parts in the head and the intermediate railway vehicles, respectively, while the masses $m_{1}$ and $m_{2}$ are, respectively, equal to those of the simplified middle parts in the original vehicle body.

(3) The mass elements $m_{1}$ and $m_{2}$ and the nodes in the sections of the residual vehicle body are connected by beam elements. It is worth noting that the stiffness of all beam elements has to be coincident with that of the simplified middle part of the vehicle body. In the implementation process, the simplified middle part of the body was extracted 


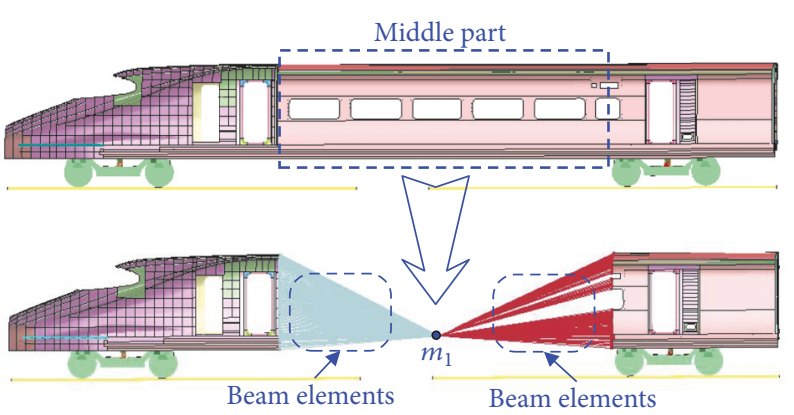

(a) Simplification process: the head vehicle

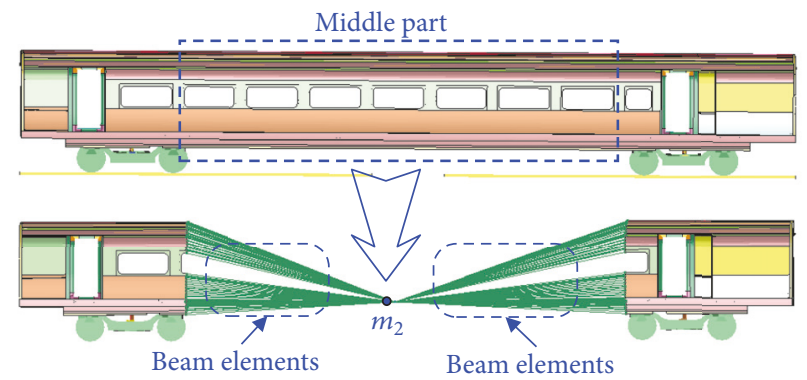

(b) Simplification process: the intermediate vehicle

FIGURE 1: Simplification processes: head and intermediate railway vehicles.

TABLE 1: Comparison of the grid scale of the whole train before, and after, simplification.

\begin{tabular}{lccc}
\hline & Number of nodes & Number of elements & Number of beam elements \\
\hline Before simplification & 4085296 & 4759820 & - \\
After simplification & 2588860 & 2930082 & 9820 \\
\hline
\end{tabular}

for the longitudinal compression simulation (one end of the structure was fixed and the other end was compressed at a quasi-static speed by an ideal rigid wall; once the plastic deformation appeared in this part, the simulation was ended immediately).

In this way, the relationship of the compression force $F$ and the compression displacement $\delta$ at the end of the structure can be calculated: $F=k \delta$. Through this relationship, the total stiffness coefficient $k$ of the simplified part of the vehicle body can be obtained. In the simplification process, the number $n$ of all beam elements was counted and then we divide the total stiffness coefficient $k$ by $n$ to obtain the stiffness coefficient $k_{1}\left(k_{1}=k / n\right)$ of each of the beam elements. In the software the stiffness coefficient $k_{1}$ is Young's modulus of each beam element.

By doing so, the grid scale of the simplified single-vehicle model can be greatly simplified. The results of the collision between the simplified head and intermediate railway vehicle and the rigid wall at an impact velocity of $10 \mathrm{~m} / \mathrm{s}$ are compared and analysed in Figures 2 and 3. As shown, the variation of the impact kinetic energy, internal energy, and impact load of the simplified vehicle body matched those of the vehicle body without simplification. The simulation time of the full and simplified head vehicle was 14 hours 30 minutes 16 seconds and 8 hours 5 minutes 29 seconds, respectively. The simulation time of the full and the simplified intermediate vehicle was 8 hours 51 minutes 47 seconds and 5 hours 28 minutes 9 seconds, respectively. The crush lengths of the head vehicle before and after simplification were 1226.2 and $1270.4 \mathrm{~mm}$. The crush lengths of the intermediate vehicle before and after simplification were 575.8 and $564.3 \mathrm{~mm}$, respectively.

(4) The head and intermediate railway vehicle are assembled based on the simplified single-vehicle model to establish the finite element model for an entire high-speed train composed of eight vehicles (Figure 4). The grid scales of the whole train before, and after, simplification are compared in Table 1. Therein, the numbers of nodes and elements in the simplified finite element model are, respectively, $63.4 \%$ and $61.6 \%$, of those of the unsimplified finite element model.

2.2. The Material Model of the Car-Body Structure. To obtain the simulation parameters for the dynamic mechanical properties and extract the LS-DYNA material cards for the numerical simulation of such thin-shell metal structures, tensile experiments were carried out on a universal material testing system (MTS) to evaluate the mechanical properties of the structures. The relationships between actual stress and strain in quasi-static loading conditions were acquired in the experiments. In addition, stress-strain curves of the samples were acquired in high-speed dynamic tensile experiments performed on a split Hopkinson bar (SHB) at different strain rates [26]. Figure 5 shows the necessary data.

The correlation curves in Figure 5 show that, with increased strain rate, the thin-walled metal had a basically constant yield strength, an apparently increasing ductility, and a slight overall strain rate effect. The aforementioned static and dynamic tensile experiments revealed that the material properties of the structure were consistent with the principles of the existing plastic kinematic simulation materials in LS-DYNA [27]. So, the material of the car-body structure was modelled as a plastic kinematic material. The constitutive relationship of the material can be described as

$$
\sigma_{y}=\left[1+\left(\frac{\dot{\varepsilon}}{C}\right)^{1 / p}\right]\left(\sigma_{0}+\beta E_{p} \varepsilon_{\text {eff }}^{p}\right),
$$

where $\sigma_{y}$ is the yield stress; $\sigma_{0}$ is the initial yield stress; $\dot{\varepsilon}$ is the strain rate; $C$ and $p$ are the strain rate parameters of Cowper Symonds; $\beta$ is the hardening parameter; $\varepsilon_{\text {eff }}^{p}$ is the effective plastic strain; $E_{p}$ is the hardening plastic modulus, $E_{p}=E_{\mathrm{tan}} E /\left(E-E_{\mathrm{tan}}\right) ; E$ is the Young's modulus; $E_{\mathrm{tan}}$ is the tangent modulus of plastic deformation. 


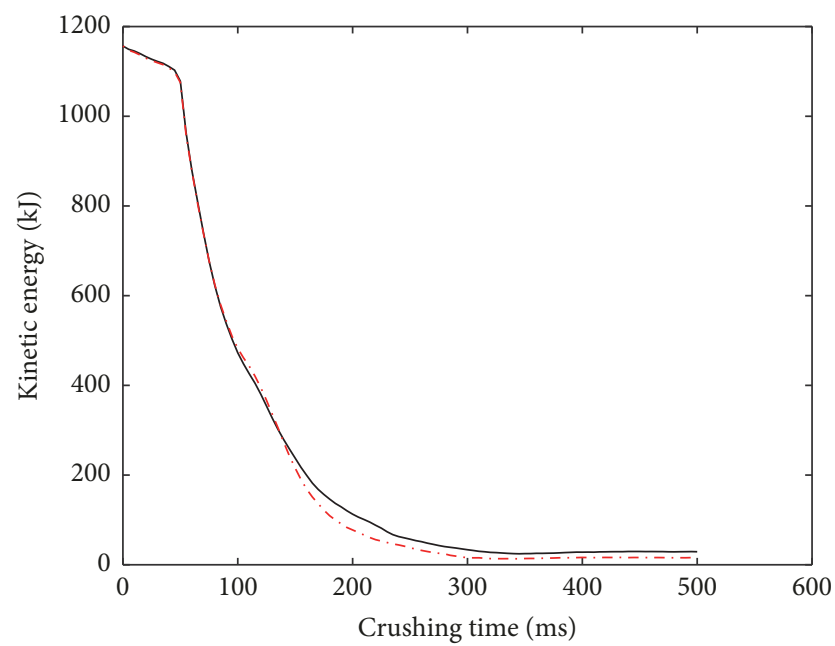

- Before simplification ...- After simplification

(a) Comparison of the kinetic energy

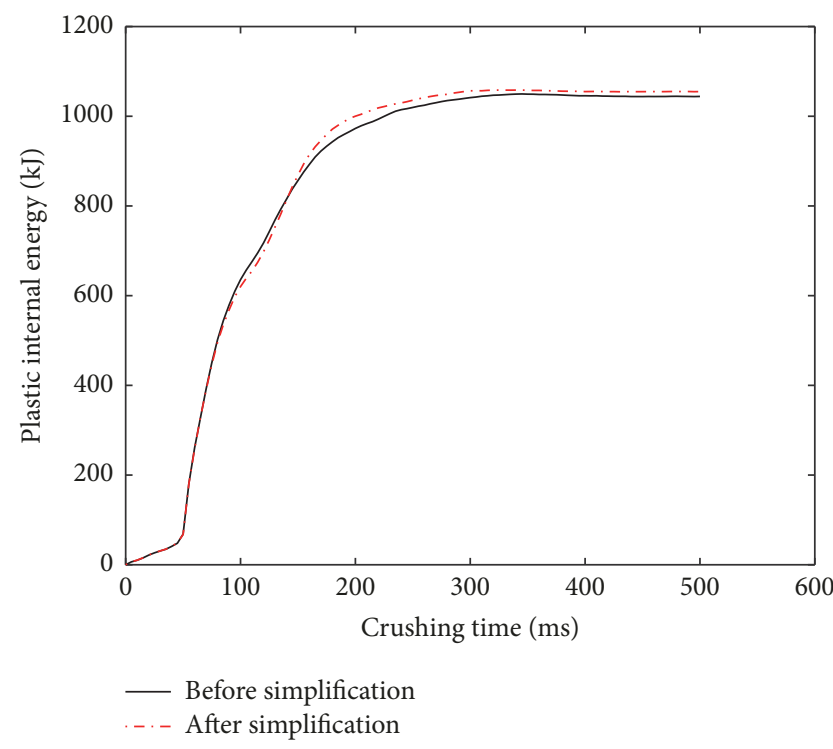

(b) Comparison of the internal energy

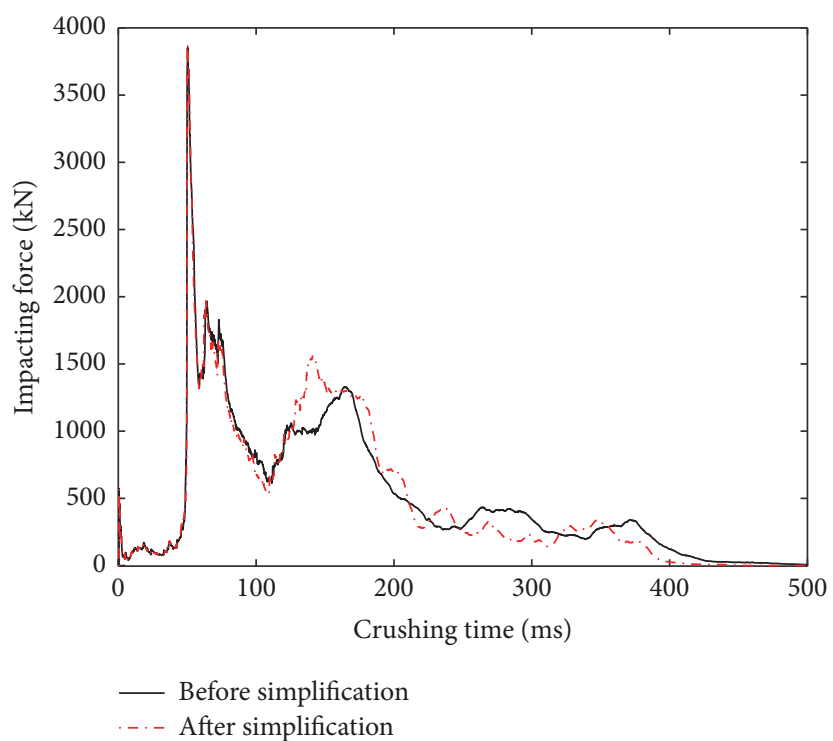

(c) Comparison of the impact force

FIGURE 2: Comparative analysis of the collision data (head vehicle) before, and after, simplification.

TABLE 2: Material parameters of the car-body structure.

\begin{tabular}{lcccccccc}
\hline Material parameters & $\rho /\left(\mathrm{kg} \cdot \mathrm{m}^{-3}\right)$ & $v$ & $E / \mathrm{GPa}$ & $\beta$ & $\sigma_{0} / \mathrm{MPa}$ & $E_{\tan } / \mathrm{MPa}$ & $C / \mathrm{s}^{-1}$ & $P$ \\
\hline Numerical value & $2.53 \times 10^{3}$ & 0.30 & 68 & 1 & 160 & 632 & $/$ \\
\hline
\end{tabular}

Based on the analysis of the experimental results for the material properties of the structure, the parameters in Table 2 were used to represent the car-body structures in collision numerical analysis.

2.3. The Collision Model of the Whole Train Set. The highspeed train investigated in this research comprises eight vehicles including four motor vehicles and four trailers. It is divided into two vehicle types, namely, the head (tail) vehicle and the intermediate railway vehicle. As demonstrated in the collision analysis of a single vehicle, when the vehicle body hits a rigid wall, large plastic deformation is only found in those parts close to the impact end, while small elastic deformation is produced in the middle parts of the vehicle body. Meanwhile, the middle part of the train is long, and if it is simulated in detail, a large number of elements are needed. 


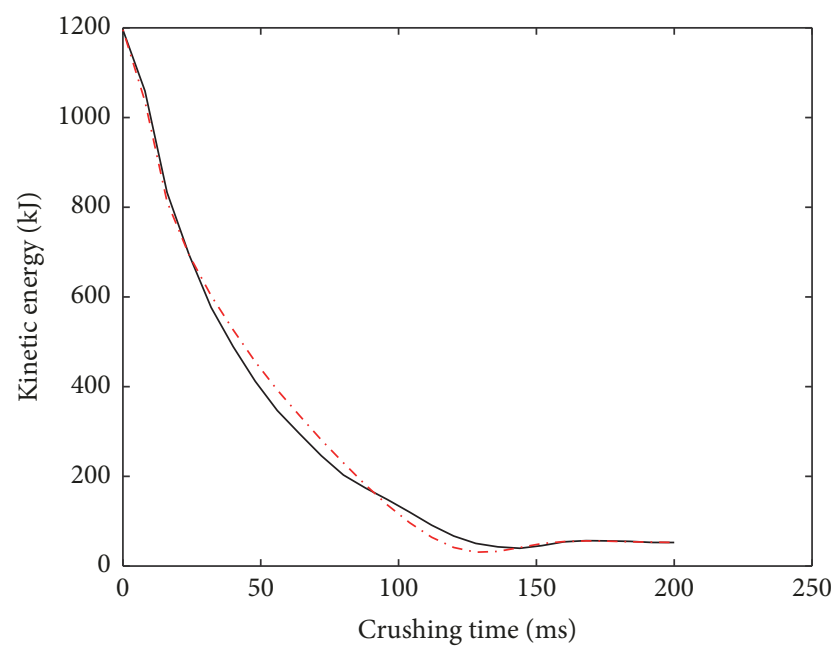

— Before simplification ...- After simplification

(a) Comparison of the kinetic energy

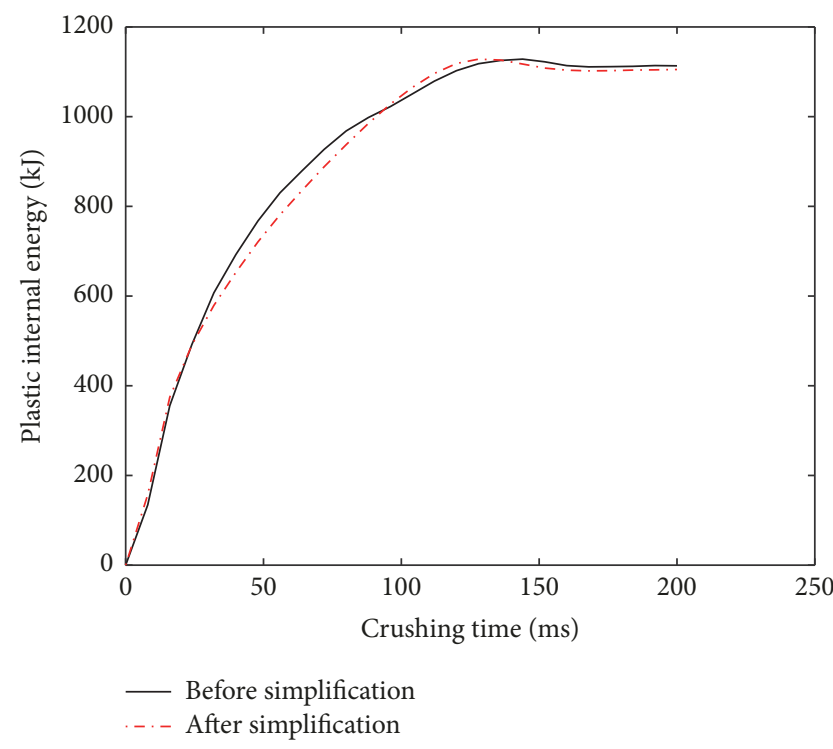

(b) Comparison of the internal energy

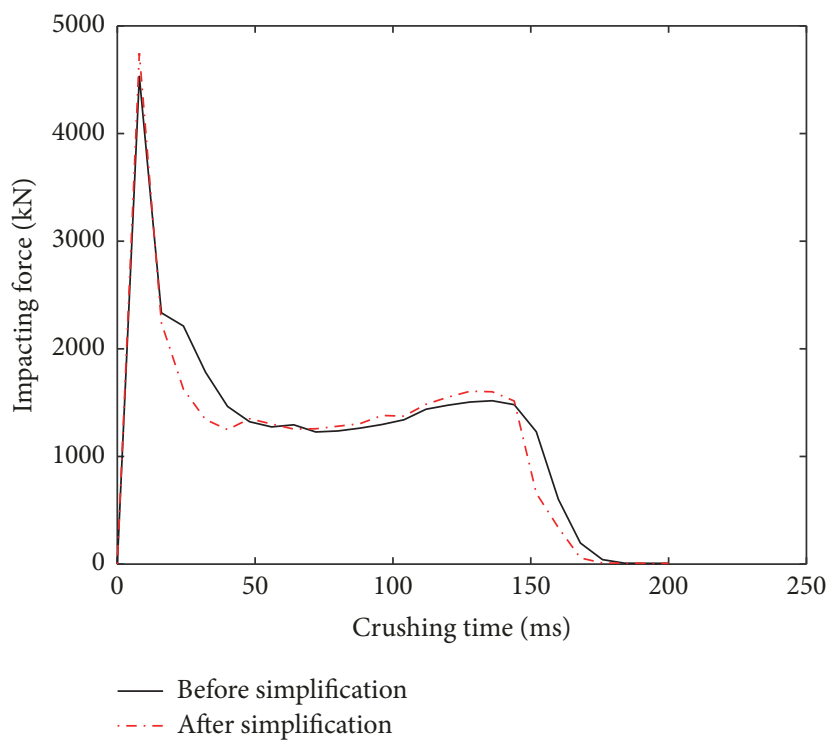

(c) Comparison of the impact force

FIGURE 3: Comparative analysis of the collision data (intermediate vehicle) before, and after, simplification.

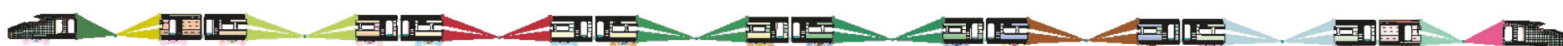

FIgURE 4: The structural model of the whole train.

To reduce the model scale and shorten computing time, the equivalent simplified models of the head and intermediate railway vehicles were built, respectively, in this research. Meanwhile, the finite element model of the whole train set was constructed based on the simplified single-vehicle model. According to the requirements of the European Norm (EN) 15227 for impact mass, impact mass is the curb weight plus $50 \%$ of the weight of the seated passengers at normal running state [28]. The bogie and the rail model were also introduced. The commonly used nonlinear spring element was employed to model the coupler draft gears between the end trunks of the vehicles to simulate the performances of the buffers in the head (Figure 6) and the intermediate railway vehicle (Figure 7), respectively.

While conducting collision analysis, a universal automatic single surface contact (ASSC) was mainly used to define 


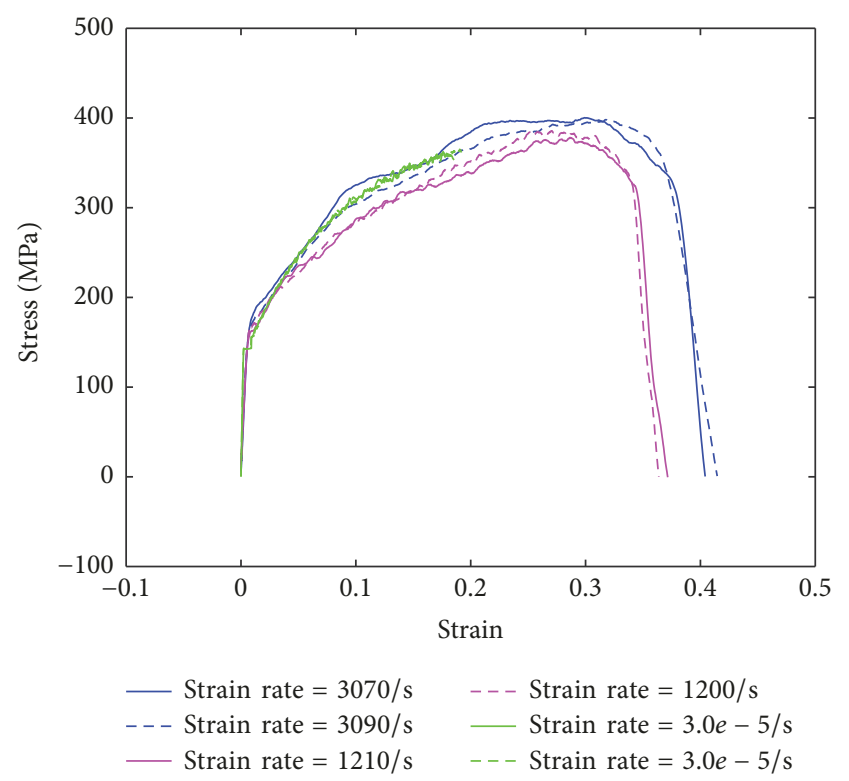

FIGURE 5: Stress-strain curves under static and dynamic loading.

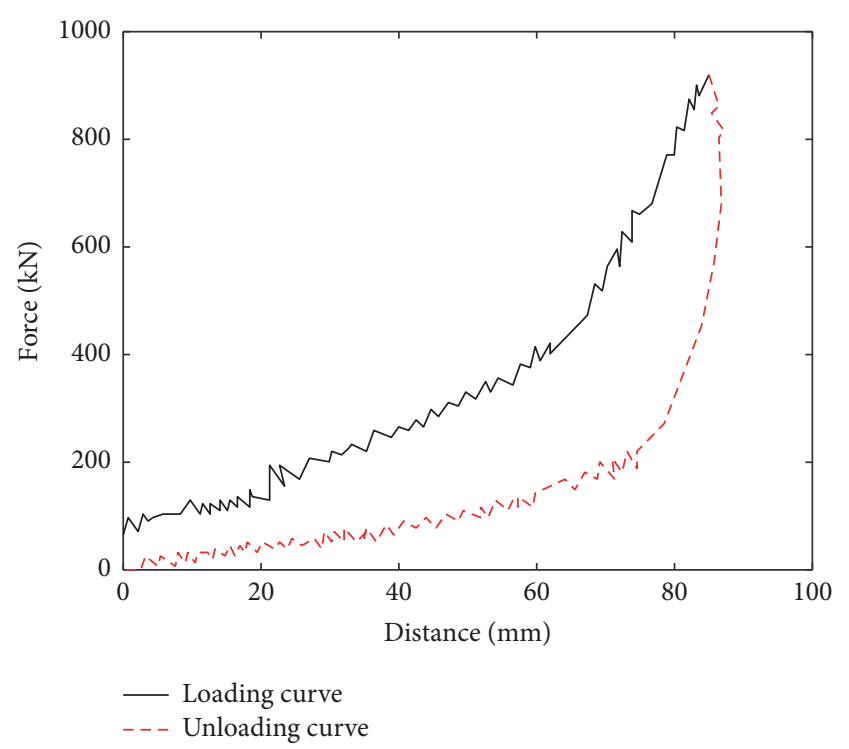

Figure 6: Performance curve of the buffer of the head vehicle.

mutual and self-contact within the inside and outside surfaces of all components of the vehicle body during collision. Meanwhile, the values of the calculation parameters including static friction coefficient, dynamic friction coefficient, and global damping coefficient were $0.3,0.2$, and 0.1 , respectively. The established finite element model of the collision between two trains is illustrated in Figure 8.

\section{Multiple-Vehicle Collision Analysis}

To study the crashworthiness of high-speed train, multiple collision cases (a train running at a certain speed collides with the same type of static train on a straight track) were used for simulation analysis (Figure 8). To save computing

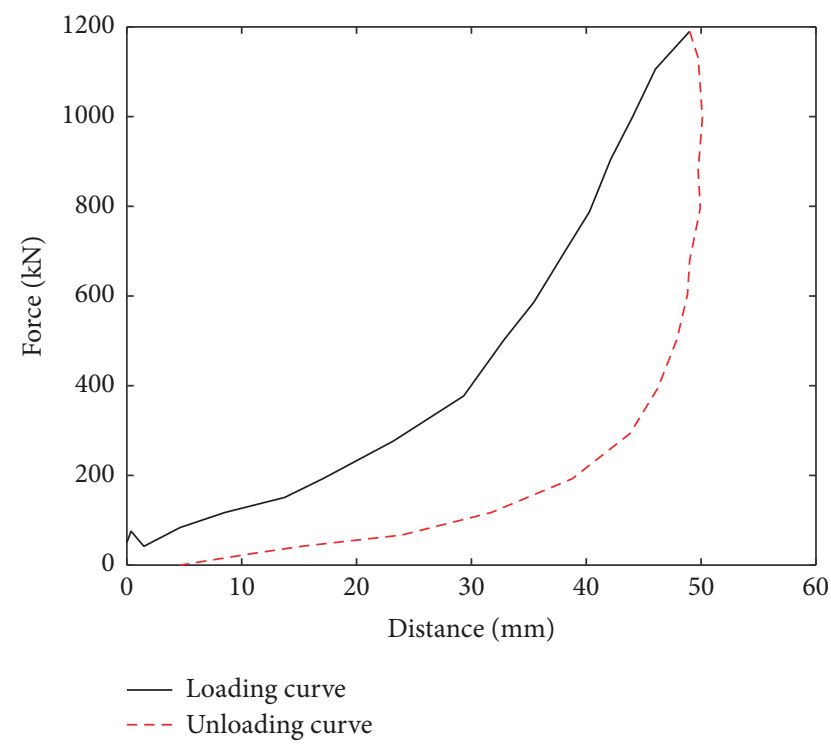

FIGURE 7: Performance curve of the buffer of the intermediate railway vehicle.

time, the equivalent simplification method introduced here was applied to the head and intermediate railway vehicles; the set of cases modelled was as follows:

Case 1: a train hits another static train at a velocity of $10 \mathrm{~km} / \mathrm{h}$;

Case 2: a train hits another static train at a velocity of $15 \mathrm{~km} / \mathrm{h}$;

Case 3: a train hits another static train at a velocity of $20 \mathrm{~km} / \mathrm{h}$;

Case 4: a train hits another static train at a velocity of $25 \mathrm{~km} / \mathrm{h}$;

Case 5: a train hits another static train unit at a velocity of $30 \mathrm{~km} / \mathrm{h}$;

Figures 9 and 10 illustrate the calculated results for each case. When the initial impact velocity is $10 \mathrm{~km} / \mathrm{h}$, the velocity variations of each vehicle in the active impact train are similar, exhibiting a gradual, sinuous reduction. Due to the small initial impact velocity, the velocities of each vehicle in the static (struck) train show little change except in the head vehicle. With increasing initial impact velocity, the velocities of each vehicle in the moving train all indicate a gradually sinuous decrease. The vehicles in the static (struck) train are firstly accelerated under the influence of the impact and then slowed down by the coupler buffer device and friction. When the impact kinetic energy is dissipated, the velocities of the two trains gradually tend to zero. Meanwhile, all vehicles experience fluctuating velocity variations, which suggests that there are secondary collisions between vehicles.

As observed in the acceleration plots of each vehicle in each train, when the initial impact velocity is $10 \mathrm{~km} / \mathrm{h}$, the acceleration of the head vehicles in the moving and static trains fluctuates. With increasing initial impact velocity, the acceleration of the vehicles in the second section of 


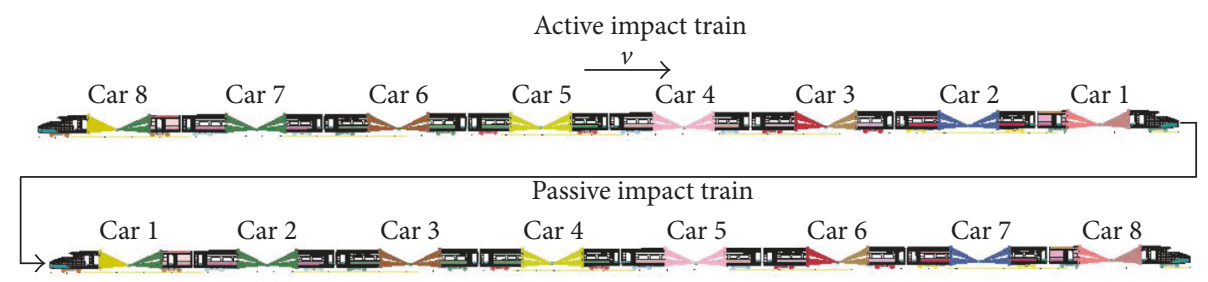

Figure 8: The collision model.
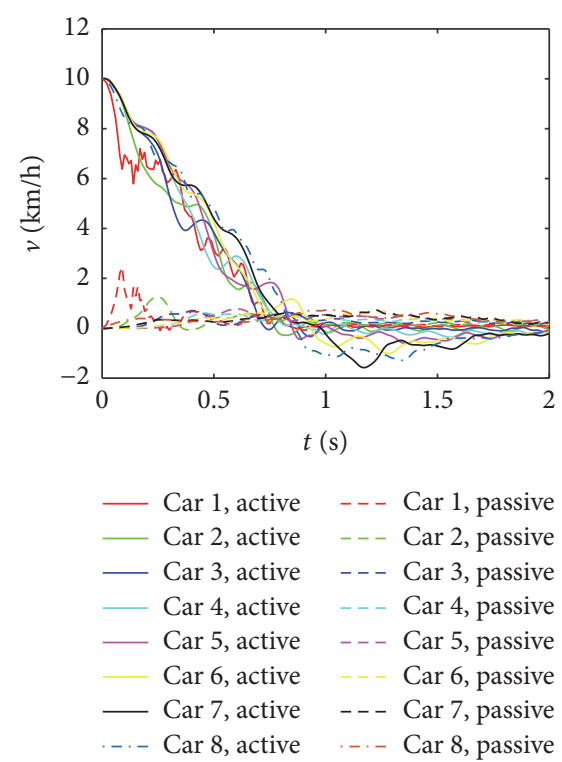

(a) Case $1(10 \mathrm{~km} / \mathrm{h})$
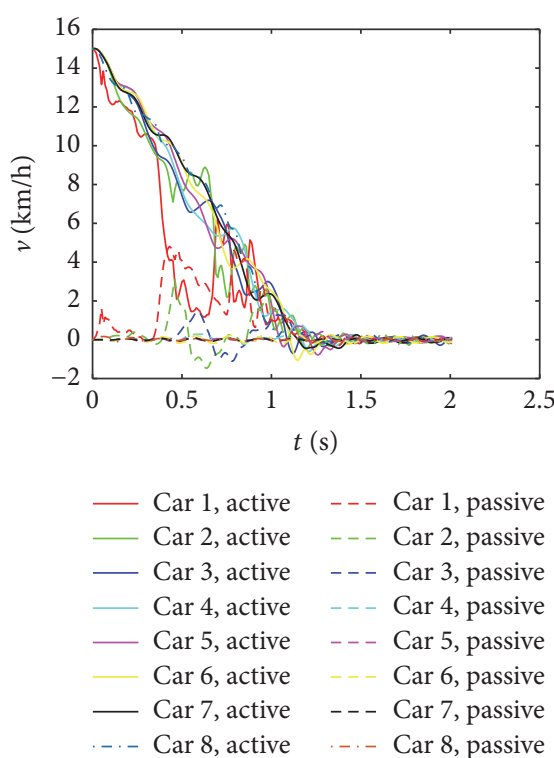

(b) Case $2(15 \mathrm{~km} / \mathrm{h})$
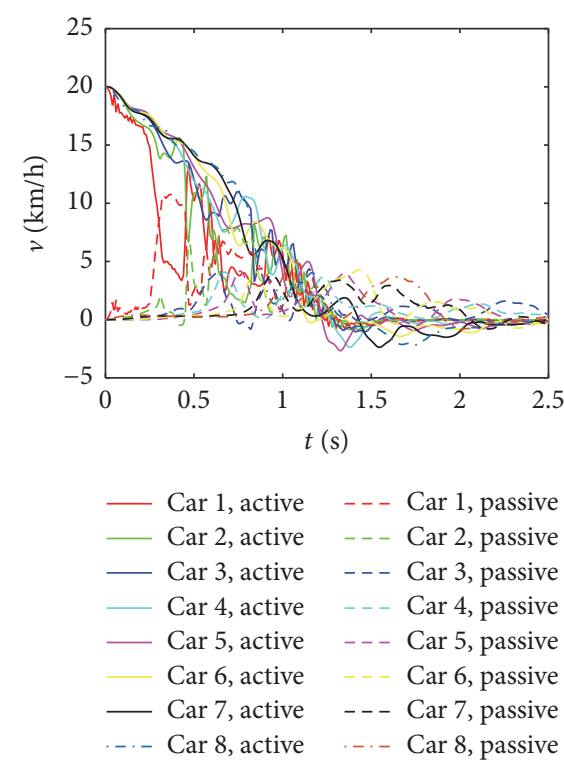

(c) Case $3(20 \mathrm{~km} / \mathrm{h})$
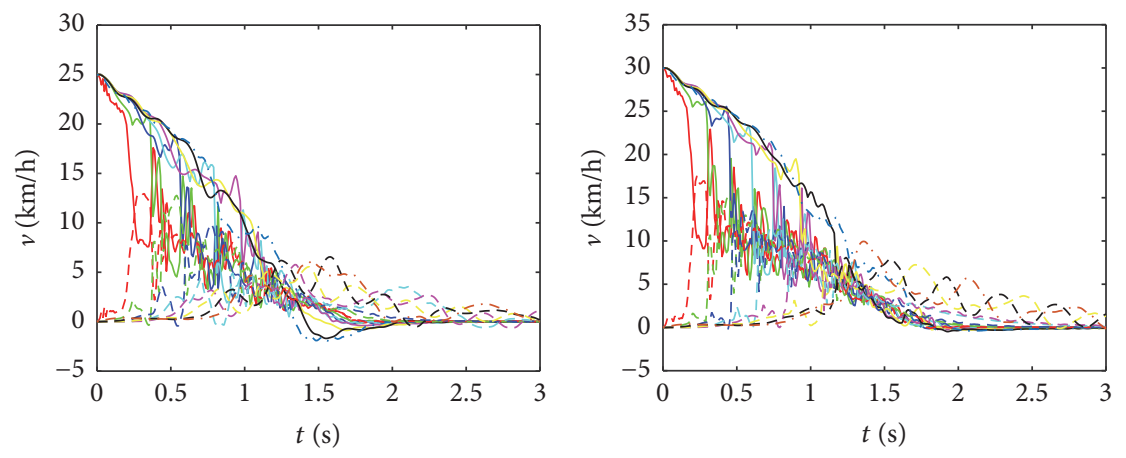

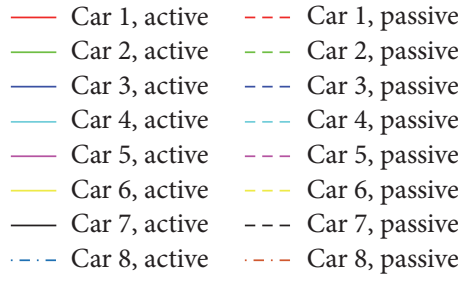

(d) Case $4(25 \mathrm{~km} / \mathrm{h})$

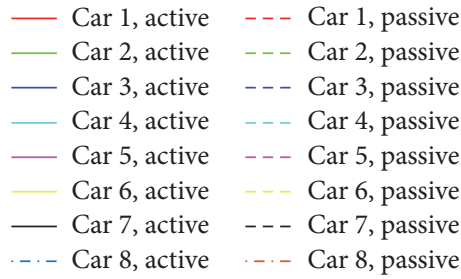

(e) Case $5(30 \mathrm{~km} / \mathrm{h})$

FIgURE 9: Velocity-time curves for all cars.

each train also fluctuates, and the peak in the acceleration curve indicates that this vehicle is colliding with an adjacent vehicle. Similarly, the fluctuating acceleration in the vehicles thereafter also suggests that multibody coupled collisions happen between vehicles. According to the requirements of EN15227, the average longitudinal acceleration/deceleration within the living space of the passengers is supposed to be no more than $5 g$ (where $g$ is the acceleration due to 

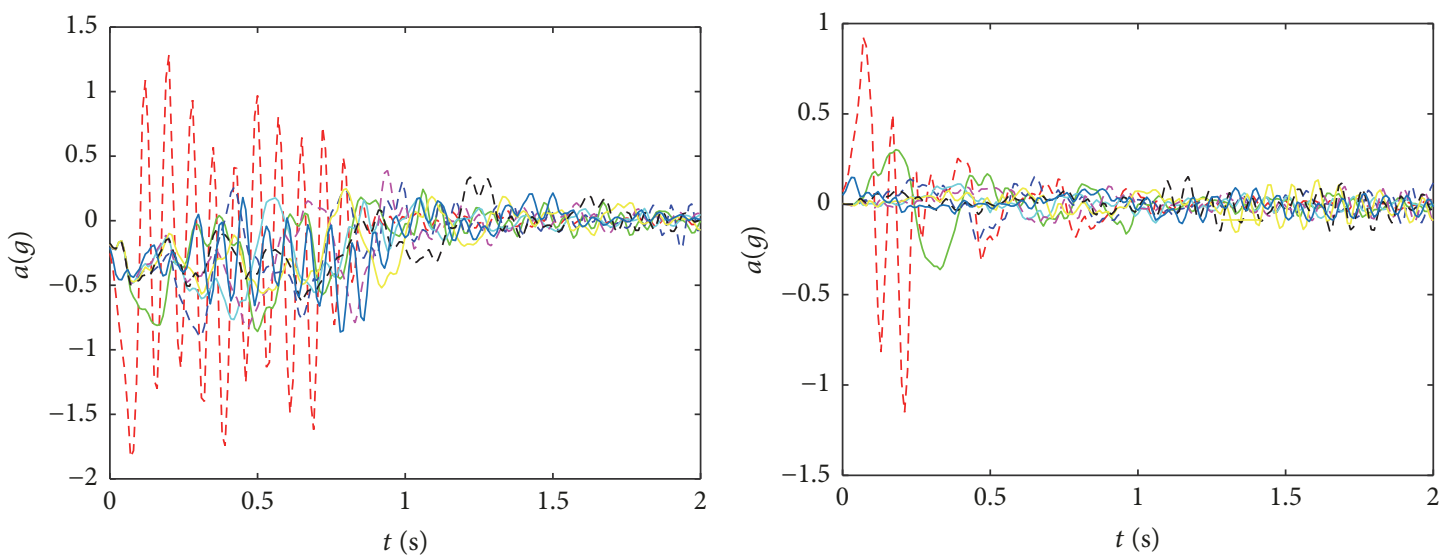

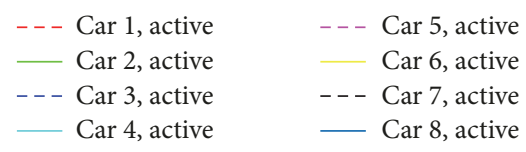

(a) Active impact cars for case 1

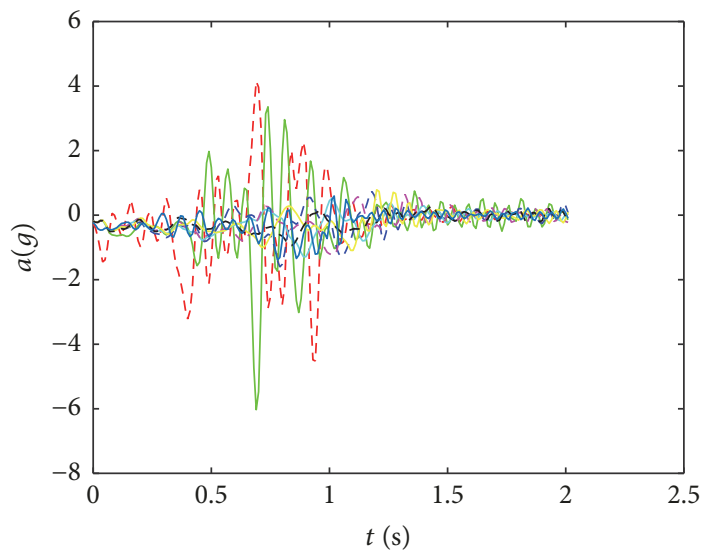

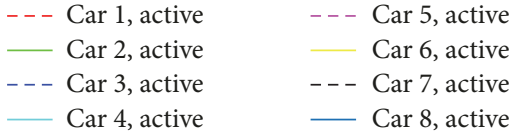

(c) Active impact cars for case 2

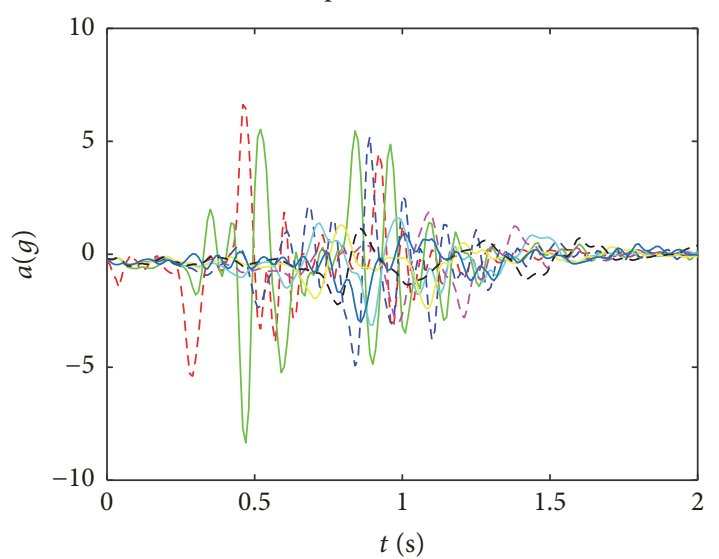

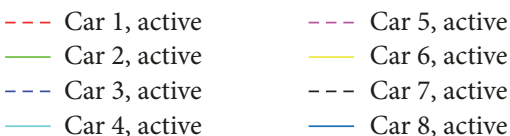

(e) Active impact cars for case 3

$\begin{array}{rr}--- \text { Car 1, passive } & -- \text { Car 5, passive } \\ - \text { - Car 2, passive } & \text { Car 6, passive } \\ -- \text { Car 3, passive } & -- \text { Car 7, passive } \\ - \text { Car 4, passive } & - \text { Car 8, passive }\end{array}$

(b) Passive impact cars for case 1
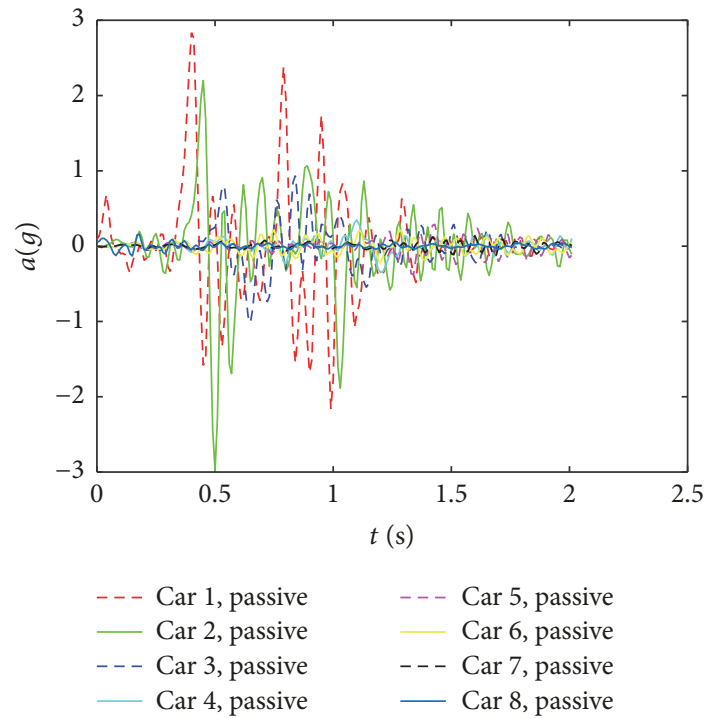

(d) Passive impact cars for case 2
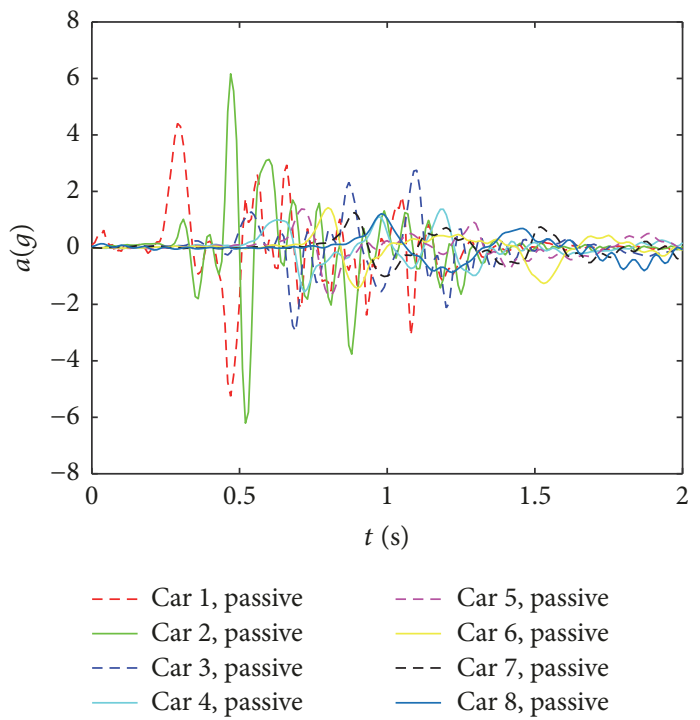

(f) Passive impact cars for case 3

Figure 10: Continued. 

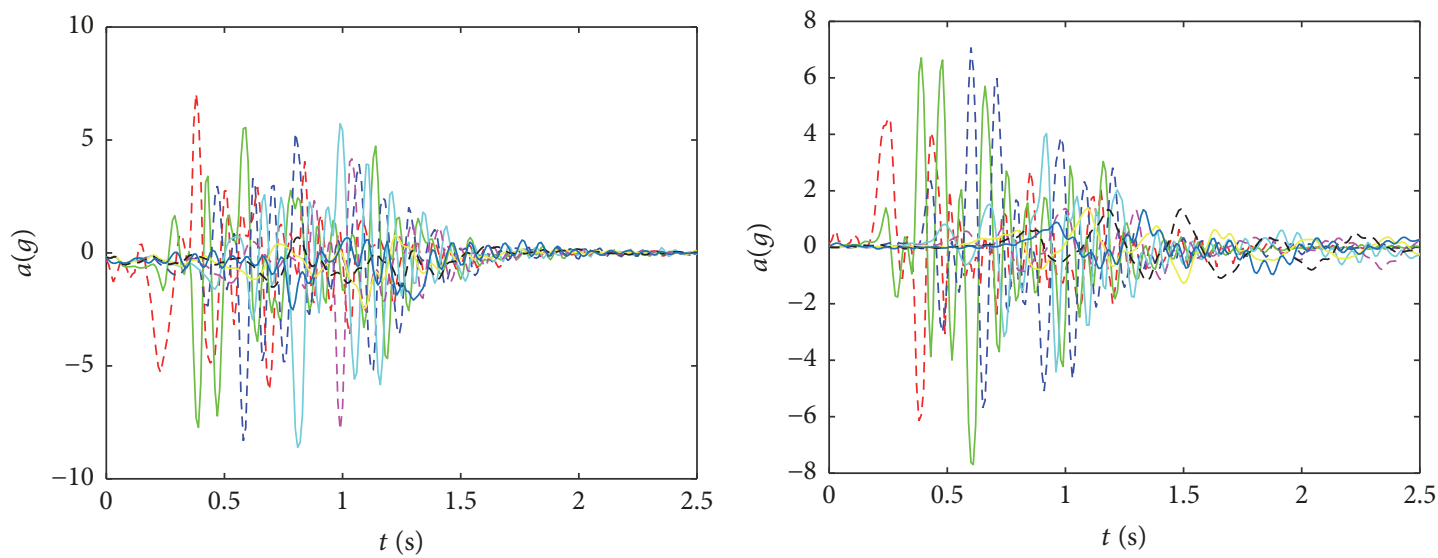

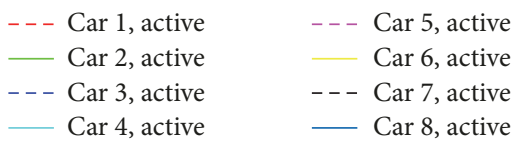

(g) Active impact cars for case 4

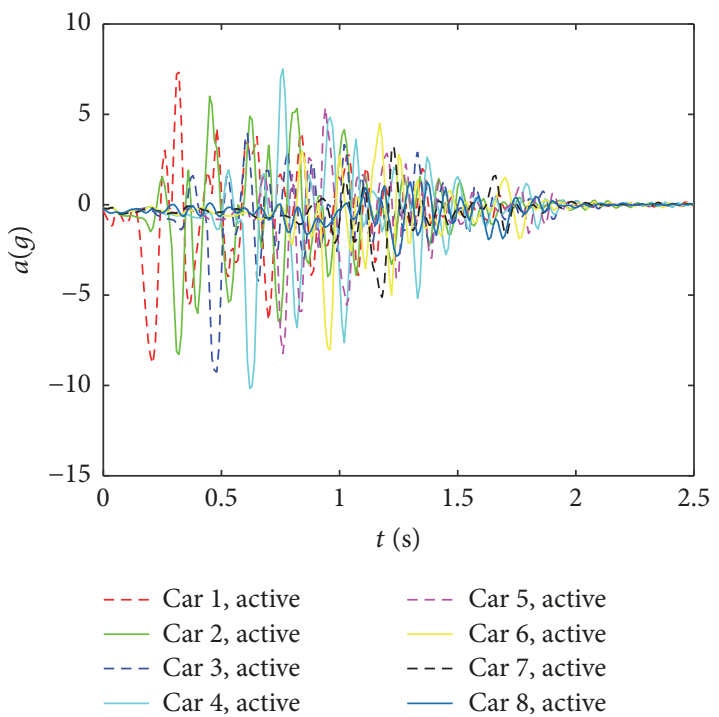

(i) Active impact cars for case 5

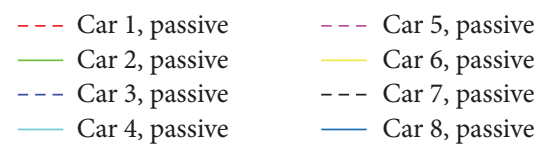

(h) Passive impact cars for case 4

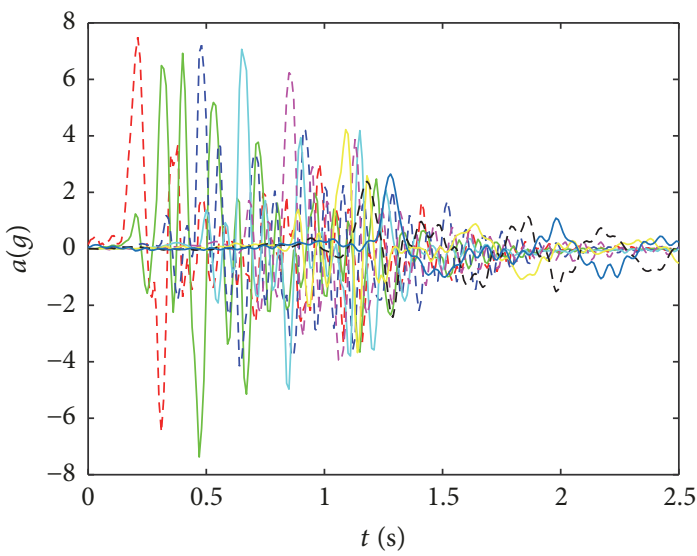

$\begin{array}{lr}\text { - - Car 1, passive } & \text { - - Car 5, passive } \\ \text { - } \text { Car 2, passive } & \text { Car 6, passive } \\ \text { - - Car 3, passive } & \text { - - Car 7, passive } \\ \text { - Car 4, passive } & \text { - Car 8, passive }\end{array}$

(j) Passive impact cars for case 5

FIGURE 10: Individual acceleration-time curve for all cars.

gravity) during a collision. When the initial impact velocity is $10 \mathrm{~km} / \mathrm{h}$, the maximum acceleration in the moving train appears in the head vehicle and is $-1.83 \mathrm{~g}$. Meanwhile, the maximum acceleration in the static (struck) train is also found in the head vehicle and is $-1.15 g$. The longitudinal acceleration/deceleration of all vehicles is reduced to below $5 g$ in this case, indicating that the acceleration/deceleration can satisfy the requirements imposed. When the initial impact velocity is $15 \mathrm{~km} / \mathrm{h}$, the peak acceleration of the vehicle in the second section of the moving train reaches a maximum of $-6.02 \mathrm{~g}$. At an initial impact velocity of $20 \mathrm{~km} / \mathrm{h}$, the peak acceleration in the head vehicle and the vehicle in the second section of the moving train is $6.63 \mathrm{~g}$ and $-8.34 \mathrm{~g}$, respectively, while that of the static (struck) train is $-5.25 \mathrm{~g}$ and $-6.21 g$ separately. With the increase of initial impact velocity, the peak acceleration of the subsequent vehicles gradually exceeds the standard value specified in EN15227.

\section{Conclusions}

(1) To overcome the large scale of the finite element model, this train is simplified by using the proposed method and as a result, less computing time was required. Meanwhile, most everyday computers may be used as the method saved storage space and greatly increased analytical efficiency.

(2) As seen in the comparative analysis of the collision between a single railway vehicle (including head and intermediate vehicles before, and after, simplification) and a rigid wall, the variations in impact 
kinetic energy, internal energy, and impact force (after simplification) matched those before simplification. Meanwhile, the finite element model of a whole highspeed train is assembled based on the simplified single-vehicle model. The numbers of nodes and elements in the simplified finite element model of the whole train are $63.4 \%$ and $61.6 \%$, respectively, of those of the unsimplified model.

(3) As to the model of the whole train simplified using the method proposed in this paper, it is more accurate than the multibody model. Meanwhile, compared with the full-size finite element model, it is more specific, accompanied with rapid computational speed, and can save a large amount of storage space.

(4) The velocity variations within each vehicle in the moving train are similar and show a gradually sinuous decrease. The vehicles in the static (struck) train firstly accelerated upon impact and then decelerated because of the coupler buffer device and friction. The velocities of the two trains tended to zero as the kinetic energy was dissipated. A fluctuating velocity was found in each vehicle during the collision. This demonstrates the presence of secondary collisions between vehicles.

(5) The peak in the acceleration curve indicates that this vehicle is colliding with an adjacent vehicle, and similarly, the fluctuating acceleration in subsequent vehicles also suggests that multibody coupled collisions occur between trains.

\section{Conflicts of Interest}

The authors declare that they have no conflicts of interest.

\section{Acknowledgments}

This research was undertaken at the Key Laboratory for Traffic Safety on Track of the Ministry of Education, Central South University, China. The authors gratefully acknowledge the support from the National Natural Science Foundation of China (Grant nos. 51775558, 51405516), the support by Innovation-Driven Project of Central South University (no. 2018CX023), and the support from the Shenghua Yu-ying Talents Program of the Central South University (Principle Investigator: Professor Suchao Xie). The author thanks Professor Ping Xu for his support in material experiments of the car-body structure.

\section{References}

[1] S. Xie and H. Zhou, "Impact characteristics of a composite energy absorbing bearing structure for railway vehicles," Composites Part B: Engineering, vol. 67, pp. 455-463, 2014.

[2] S. Chatterjee and J. F. Carney III, "Passenger train crashworthiness - Primary collisions," Transportation Research Record, vol. 18, no. 1531, pp. 1-12, 1996.

[3] S. Chatterjee and J. F. Carney III, "Passenger train crashworthiness - Secondary collisions," Transportation Research Record, vol. 18, no. 1531, pp. 13-19, 1996.
[4] C. Baykasoglu, E. Sunbuloglu, E. Bozdag, F. Aruk, T. Toprak, and A. Mugan, "Railroad passenger car collision analysis and modifications for improved crashworthiness," International Journal of Crashworthiness, vol. 16, no. 3, pp. 319-329, 2011.

[5] P. Hosseini-Tehrani and A. Nankali, "Study on characteristics of a crashworthy high-speed train nose," International Journal of Crashworthiness, vol. 15, no. 2, pp. 161-173, 2010.

[6] H. Shao, P. Xu, S. Yao, Y. Peng, R. Li, and S. Zhao, "Improved multibody dynamics for investigating energy dissipation in train collisions based on scaling laws," Shock and Vibration, vol. 2016, Article ID 3084052, 11 pages, 2016.

[7] H. Dong and Q. Wang, "On the critical speed, supercritical bifurcation, and stability problems of certain type of high-speed rail vehicle," Shock and Vibration, vol. 2017, Article ID 1526373, pp. 1-9, 2017.

[8] Y. Yang, G. Zhao, D. Ma, and X. Xu, "Mode calculation and testing of a car body in white," Shock and Vibration, vol. 18, no. 1-2, pp. 289-298, 2011.

[9] S. Xie and H. Zhou, "Research on the crashworthy structures of subway vehicles," International Journal of Crashworthiness, vol. 19, no. 6, pp. 555-566, 2014.

[10] C. Baykasoglu, E. Sunbuloglu, S. E. Bozdag, F. Aruk, T. Toprak, and A. Mugan, "Crash and structural analyses of an aluminium railroad passenger car," International Journal of Crashworthiness, vol. 17, no. 5, pp. 519-528, 2012.

[11] G. J. Gao and H. Q. Tian, “Train's crashworthiness design and collision analysis," International Journal of Crashworthiness, vol. 12, no. 1, pp. 21-28, 2007.

[12] S. C. Xie and H. Q. Tian, "Dynamic simulation of railway vehicle occupants under secondary impact," Vehicle System Dynamics, vol. 51, no. 12, pp. 1803-1817, 2013.

[13] H.-S. Han and J.-S. Koo, "Simulation of train crashes in three dimensions," Vehicle System Dynamics, vol. 40, no. 6, pp. 435450, 2003.

[14] J. W. Simons and S. W. Kirkpatrick, "High-speed passenger train crashworthiness and occupant survivability," International Journal of Crashworthiness, vol. 4, no. 2, pp. 121-132, 1999.

[15] J. F. Milho, J. A. C. Ambrósio, and M. F. O. S. Pereira, "Validated multibody model for train crash analysis," International Journal of Crashworthiness, vol. 8, no. 4, pp. 339-352, 2003.

[16] J. F. Milho, J. A. C. Ambrósio, and M. F. O. S. Pereira, "A multibody methodology for the design of anti-climber devices for train crashworthiness simulation," International Journal of Crashworthiness, vol. 7, no. 1, pp. 7-20, 2002.

[17] S. W. Kirkpatrick, M. Schroeder, and J. W. Simons, "Evaluation of passenger rail vehicle crashworthiness," International Journal of Crashworthiness, vol. 6, no. 1, pp. 95-106, 2001.

[18] G. Lu, "Energy absorption requirement for crashworthy vehicles, part F," Journal of Rail and Rapid Transit, vol. 216, no. 1, pp. 31-39, 2002.

[19] G. Lu, "Collision behaviour of crashworthy vehicles in rakes," in Proceedings of the Institution of Mechanical Engineers, Part F: Journal of Rail and Rapid Transit, vol. 213, pp. 143-160, 1999.

[20] X. Suchao and T. Hongqi, "Influencing factors and sensitivity analysis of occupant impact injury in passenger compartment," Traffic Injury Prevention, vol. 14, no. 8, pp. 816-822, 2013.

[21] Q. Yan, Z. Deng, Y. Zhang, and W. Yang, "Failure characteristics of joint bolts in shield tunnels subjected to impact loads from a derailed train," Shock and Vibration, vol. 2017, Article ID 2829783, 9 pages, 2017. 
[22] Z. H. Zhong, Finite Element Procedures for Contact-Impact Problems, University Press, Oxford, UK, 1993.

[23] A. P. Meran, C. Baykasoglu, A. Mugan, and T. Toprak, "Development of a design for a crash energy management system for use in a railway passenger car," Proceedings of the Institution of Mechanical Engineers, Part F: Journal of Rail and Rapid Transit, vol. 230, no. 1, pp. 206-219, 2016.

[24] Z. Tang, Y. Zhu, Y. Nie et al., "Data-driven train set crash dynamics simulation," Vehicle System Dynamics, vol. 55, no. 2, pp. 149-167, 2017.

[25] S. Afazov, W. Denmark, and A. Yaghi, "Modelling aspects of the design of railway vehicle structures and their crashworthiness," Proceedings of the Institution of Mechanical Engineers, Part F: Journal of Rail and Rapid Transit, vol. 230, no. 6, pp. 1575-1589, 2016.

[26] S. Xie, X. Liang, H. Zhou, and J. Li, "Crashworthiness optimisation of the front-end structure of the lead car of a high-speed train," Structural and Multidisciplinary Optimization, vol. 53, no. 2, pp. 339-347, 2016.

[27] J. O. Hallquist, LS-DYNA Theory Manual, Livermore Software Technology Corporation, Calif, USA, 2006.

[28] UK., "Railway applications-Crashworthiness requirements for railway vehicle bodies. EN 15227:2008. British: Standards Policy and Strategy Committee; 2009". 


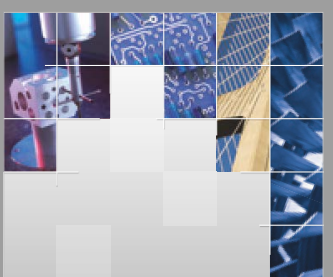

\section{Enfincering}
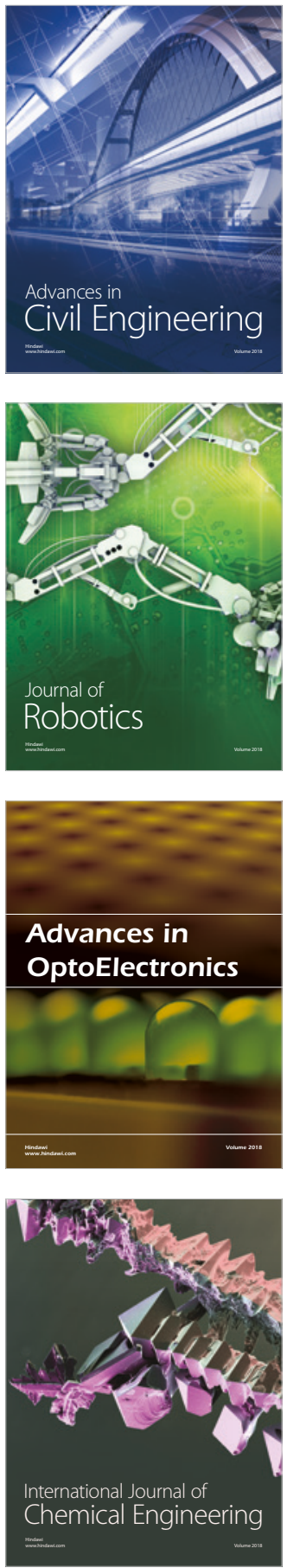

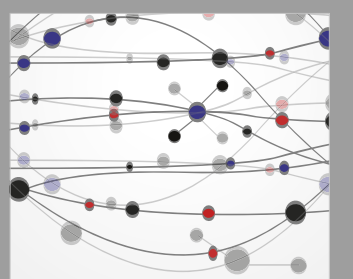

\section{Rotating \\ Machinery}

The Scientific World Journal

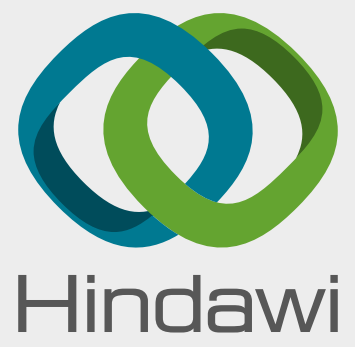

Submit your manuscripts at

www.hindawi.com
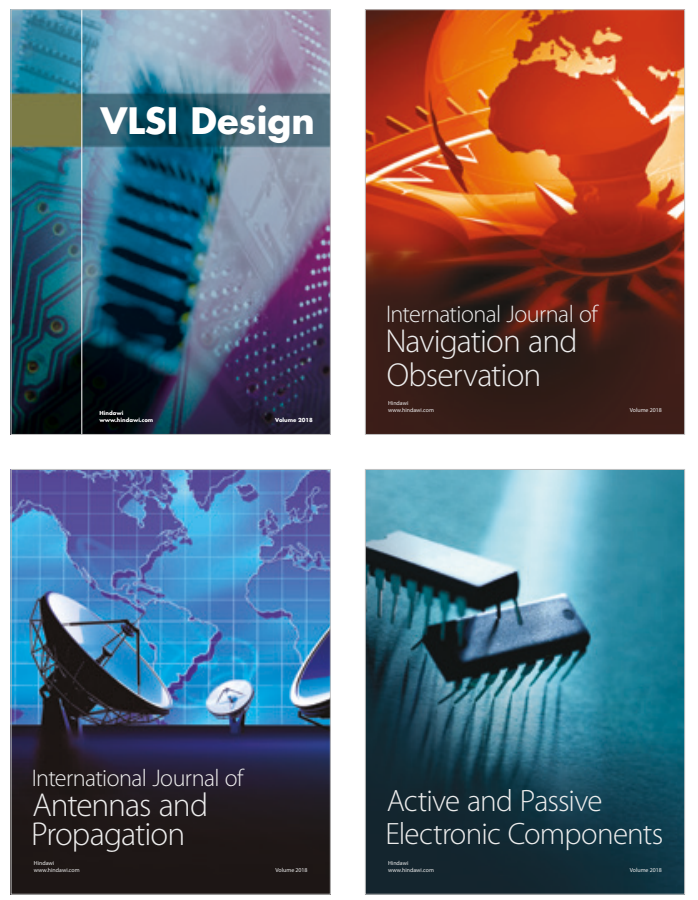
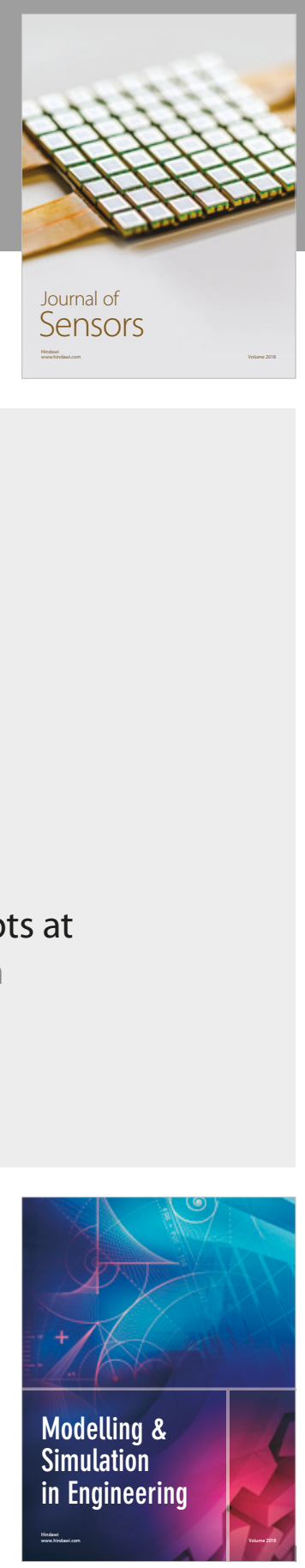

\section{Advances \\ Multimedia}
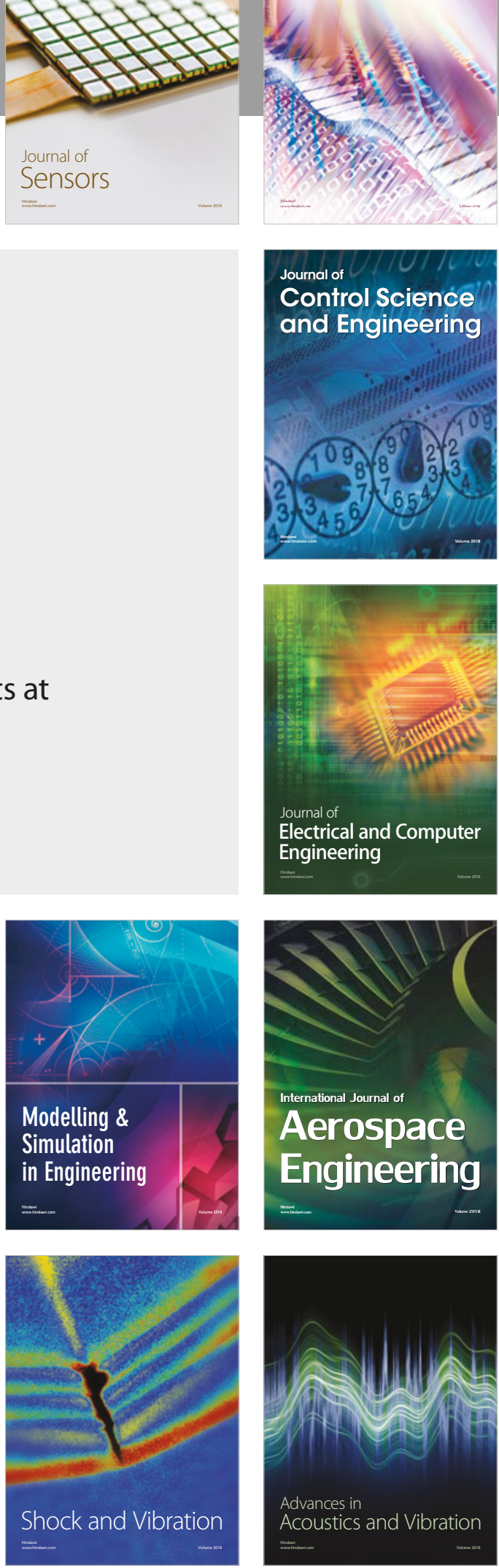